\title{
Effects of Repeated Ovarian Stimulation on Ovarian Function and Aging in Mice
}

\author{
Jihye Whang, Cheyoung Ahn, Soohyun Kim, Eunji Seok, Yunjeong Yang, \\ Goeun Han, Haeun Jo, and ${ }^{\dagger}$ Hyunwon Yang
}

Dept. of Bioenvironmental Technology, College of Natural Sciences, Seoul Women's University, Seoul 01797, Korea

Received: September 29, 2021

Revised: November 21, 2021

Accepted: December 16, 2021

${ }^{\dagger}$ Corresponding author

Hyunwon Yang

Dept. of Bioenvironmental Technology,

Seoul Women's University, Seoul 01797,

Korea.

Tel: +82-2-970-5662

Fax: +82-2-970-5974

E-mail: hwyang@swu.ac.kr

Copyright $\odot 2021$ The Korean Society of Developmental Biology.

This is an Open Access article distributed under the terms of the Creative Commons Attribution Non-Commercial License (http://creativecommons.org/licenses/ by-nc/4.0/) which permits unrestricted non-commercial use, distribution, and reproduction in any medium, provided the original work is properly cited.

ORCID

Jihye Whang

https://orcid.org/0000-0002-1487-3481

Cheyoung Ahn

https://orcid.org/0000-0001-5945-4080

Soohyun Kim

https://orcid.org/0000-0002-0873-4899

Eunji Seok

https://orcid.org/0000-0002-3988-6270

Yunjeong Yang

https://orcid.org/0000-0002-8856-0699

Goeun Han

https://orcid.org/0000-0001-7701-3342

Haeun Jo

https://orcid.org/0000-0002-6704-1638

Hyunwon Yang

https://orcid.org/0000-0002-3649-647X

Conflict of interests

The authors declare no potential conflict of interest.

Acknowledgements

This work was supported by a special research grant from Seoul Women's

\section{Abstract}

Controlled ovarian hyperstimulation $(\mathrm{COH})$ is routinely used in the in vitro fertilization and embryo transfer (IVF-ET) cycles to increase the number of retrieved mature oocytes. However, the relationship between repeated $\mathrm{COH}$ and ovarian function is still controversial. Therefore, we investigated whether repeated ovarian stimulation affects ovarian aging and function, including follicular development, autophagy, and apoptosis in follicles. Ovarian hyperstimulation in mice was induced by intraperitoneal injection with pregnant mare serum gonadotropin (PMSG) and human chorionic gonadotropin (hCG). Mice subjected to ovarian stimulation once were used as a control group and 10 times as an experimental group. Repeated injections with PMSG and hCG significantly reduced the number of primary follicles compared to a single injection. The number of secondary and antral follicles increased slightly, while the number of corpus luteum increased significantly with repeated injections. On the other hand, repeated injections did not affect apoptosis in follicles associated with follicular atresia. The expression of autophagy-related genes Atg5, Atg12, LC3B, and Beclin1, cell proliferation-related genes mTOR, apoptosis-related genes Fas, and FasL was not significantly different between the two groups. In addition, the expression of the agingrelated genes Dnmt1, Dnmt3a, and $A M H$ were also not significantly different. In this study, we demonstrated that repeated ovarian stimulation in mice affects follicular development, but not autophagy, apoptosis, aging in ovary. These results suggest that repetition of $\mathrm{COH}$ in the IVF-ET cycle may not result in ovarian aging, such as a decrease in ovarian reserve in adult women.

Keywords: Apoptosis, Autophagy, In vitro fertilization and embryo transfer (IVF-ET), Ovarian function, Repeated ovarian stimulation

\section{INTRODUCTION}

In vitro fertilization-embryo transfer (IVF-ET) is a series of processes in which fertilization that occurs inside a woman's body is artificially made outside the human body, and then an embryo is transferred to the woman to achieve pregnancy. In IVF-ET process, induction of superovulation of eggs is the first step that must be preceded for collecting mature eggs from women. Artificial superovulation is induced through gonadotropin hormone injection; a process called controlled ovarian 
University (2021).

\section{Authors' contributions}

Conceptualization: Yang $\mathrm{H}$.

Data curation: Whang J, Yang $\mathrm{H}$.

Formal analysis: $Y a n g \mathrm{H}$.

Methodology: Yang Y, Han G, Jo H.

Software: Yang $\mathrm{H}$.

Validation: Whang J, Yang $\mathrm{H}$.

Investigation: Whang J, Ahn C, Kim S, Seok E.

Writing-original draft: Whang $J$.

Writing-review \& editing: Yang $\mathrm{H}$.

\section{Ethics approval}

Animal care and experimental procedures were approved by the Institutional Animal Care and Use Committee at the Seoul Women's University in accordance with guidelines established by the Korea Food and Drug Administration. hyperstimulation $(\mathrm{COH})$. As superovulation promoters, follicle stimulating hormone (FSH), human menopausal gonadotropin (hMG), and human chorionic gonadotropin (hCG) are mainly used ( $\mathrm{Li}$ et al., 2015). FSH and hMG promote the growth of immature oocytes before ovulation and induce the growth of several dominant follicles during the ovulation cycle (Macklon et al., 2006). hCG stimulates ovulation and luteinization and regulates angiogenesis at the implantation site in the uterus (Christenson \& Stouffer, 1997).

Gonadotropin regulates follicle development and function during the ovarian cycle. Inappropriate gonadotropin stimulation inhibits the growth of follicles and induces follicular atresia. Follicular atresia is a recycling process that breaks down and degenerates non-dominant follicles to provide nutrients to the dominant follicle to ovulate (Baker, 1963). It is an essential physiological process that regulates follicle selection to ensure high-quality oocytes ovulate. In women, drastic changes in ovarian levels occur, and the rate of follicular atresia increases due to changes in steroid hormone levels during menopause. The most significant change when steroid hormone levels are out of balance is a sharp decrease in the number of primordial follicles. The rate of decline of the follicular pool increases with age due to the increase in the rate and extent of follicular atresia activation (Hansen et al., 2008).

It is known that the probability of successful pregnancy with one IVF-ET procedure is low at about 30\% (Santos et al., 2010). Therefore, infertile couples repeat the $\mathrm{COH}$ process several times to increase their chances of a successful pregnancy. However, it has been reported that repeated administration of high doses of exogenous gonadotropin leads to complications such as ovarian hyperstimulation syndrome (OHSS) (Jacobs \& Agrawal, 1998). Therefore, this complication can easily occur if the ovaries are overstimulated by gonadotropins during IVF-ET (Griesinger, 2010). On the other hand, repeating the $\mathrm{COH}$ process does not affect oocyte recovery, implantation, or pregnancy success rates (Carigara et al., 2001; Acevedo et al., 2006).

However, it is not yet clear whether repeated administration of high-dose exogenous gonadotropins induces affects ovarian function and aging. Therefore, in this study, Therefore, in this study, pregnant mare serum gonadotropin (PMSG) and hCG were injected 10 times to induce repeated ovarian stimulation in mice, and follicular development and ovarian apoptosis were investigated. In addition, expression changes of apoptosis-related genes, autophagy-related genes, and aging-related genes were evaluated.

\section{MATERIALS AND METHODS}

\section{Animals}

Eight-week-old female ICR mice were purchased from Koatech (Pyeongtaek, Korea) and housed in groups of six per cage under controlled illumination (12:12 h light/dark cycle, lights on/ off: $6 \mathrm{~h} / 18 \mathrm{~h})$ and temperature $\left(22 \pm 2^{\circ} \mathrm{C}\right)$. Animals were fed a standard rodent diet and tap water ad libitum. Animal care and experimental procedures were approved by the Institutional Animal Care and Use Committee at the Seoul Women's University in accordance with guidelines established by the Korea Food and Drug Administration.

\section{Experimental protocols and hormonal treatments}

Mice were divided into two groups $(\mathrm{n}=6)$. Mice were administered 5 IU of PMSG intraperitoneally, followed by 5 IU hCG 48 hours later. As a once-stimulation (OS) group, mice were injected with PBS nine times at intervals of one week, and then PMSG+hCG was injected once. As a repeated stimulation (RS) group, mice were injected with $\mathrm{PMSG}+\mathrm{hCG}$ ten times at 
one-week intervals. Three weeks after the last hCG injection, mice were sacrificed, and ovaries were obtained.

\section{Smear test}

A smear test was performed to determine the estrous cycle of mice. The tail of the mouse was raised to reveal its vagina. Vaginal cells are flushed by gently introducing a small amount of PBS using a spoid. A drop of PBS flushed by the spoid was smeared on the slide. The slides were observed under a light microscope, and the estrous cycle was evaluated according to the shape of the vaginal cells.

\section{Hematoxylin \& eosin staining}

The ovaries were fixed in $4 \%$ paraformaldehyde, and then rinsed in ethanol series. After embedding in a paraffin block, the tissue blocks were cut into $10 \mu \mathrm{m}$ sections. The tissue blocks were deparaffinized and rehydrated by sequentially treating xylene and ethanol, and washed with PBS. Tissue slides were stained with Harris' Hematoxylin (Muto Pure Chemicals, Tokyo, Japan) and eosin Y solution (Sigma-Aldrich, St. Louis, MO, USA). All stained ovarian sections were observed under an optical microscope (YS100, Nikon, Tokyo, Japan).

\section{TUNEL assay}

Apoptosis in the ovaries was assessed by TUNEL assay, which was performed by In Situ Cell Death Detection kit (Roche, Basel, Switzerland). Paraffin sections of ovaries were deparaffinized and washed twice with PBS and then stained with a TUNEL reaction mixture. After washing twice with PBS, the sections were counter-stained with DAPI. After washing twice with PBS, the stained sections were mounted with anti-fade mounting solution (Vectashield, Burlingame, CA, USA) and observed under a fluorescence microscope (Zeiss, Oberkochen, Germany).

\section{RNA extraction and quantitative reverse transcription polymerase chain reaction (qRT- PCR)}

The ovaries were homogenized with RNA isoplus (TaKaRa Bio, Shiga, Japen). After chloroform extraction and isopropyl alcohol precipitation, RNA was dissolved in RNase-free DEPC (TaKa$\mathrm{Ra}$ Bio) solution. The RNA concentrations were measured with the Nano-drop (Thermo Fisher Scientific, Waltham, MA, USA). First-strand cDNA synthesis was performed using the extracted RNA and oligo dT, followed by the double-strand synthesis in RT buffer (Invitrogen, Carlsbad, CA, USA) with dNTP (Bio Basic, Markham, ON, Canada) and RTase (Invitrogen). qRT-PCR was performed in a buffer solution containing template cDNA, SYBR Green (Enzynomics, Daejeon, Korea), and each primer. Primer pairs (Bioneer, Daejeon, Korea) were as follows; $18 S$ (Forward 5'-GTCTGTGATGCCCTTAGATG-3', Reverse 5'-AGCTTATGACCCGCACTTAC-3'), AMH (Forward 5'-CCACACCTCTCTCCACTGGTA-3', Reverse 5'-GGCACAAAGGTTCAGGGGG-3'), Atg5 (Forward 5'-ACTGCAGAATGACCACGACG-3', Reverse 5'-AGATCTCCAAGTGTGTGCAGC-3'), Atg12 (Forward 5'-CACACATGGCAGCACTCCTA-3', Reverse 5'-TTCCCCCAGAGGTGAGACAA-3'), Beclin1 (Forward 5'-TACCTGACCTGTTCTTTTCAGCA-3', Reverse 5'-GTAGCCCTCAGTGCCTCATC-3'), LC3B (Forward 5'-ACCAAGATCCCAGTGATTATAGAGC-3', Reverse 5'-CATGTTCACGTGGTCAGGCA-3'), Dnmt1 (Forward 5'-CGGGCTGTGCTTCCTGTC-3', Reverse 5'-TCCCTCAAGCTCCCAGTCAA-3'), Dnmt3a (Forward 5'-CTGAGCTGTACTGCAGAGGGG-3', Reverse 5'-TGGTTCTCTTCCACAGCATTCA-3'), mTOR (Forward 5'-TCCTGTTACCTCACCCGTCC-3', Reverse 5'-AGTTTCAGCATCGTGGGGTC-3'), Fas (Forward 5'-CTG- 
CGATTCTCCTGGCTGTGAA-3', Reverse 5'-CAACAACCATAGGCGATTTCTGG-3'), FasL (Forward 5'- TCCGTGAGTTCACCAACCAA-3', and Reverse 5'-TGAGTGGGGGTTCCCTGTTA-3'). The optimum temperature cycling protocol was determined to be $95^{\circ} \mathrm{C}$ for $10 \mathrm{~s}, 60^{\circ} \mathrm{C}$ for $10 \mathrm{~s}$ and $72^{\circ} \mathrm{C}$ for $10 \mathrm{~s}$ using the Light Cycler 480 Real-time PCR System (Roche, Manheim, Germany).

\section{RESULTS}

\section{Effect of repeated ovarian stimulation on estrous cycle}

The estrous cycle in mice was examined through a smear test. Each stage of the estrous cycle was determined according to the composition and morphology of vaginal epithelial cells and the number of erythrocytes (Chari et al., 2020). In the OS group, keratinized epithelial cells and a small number of red blood cells observed at the estrous and metestrous stages were observed. In the RS group, a small number of keratinized epithelial cells and a large number of erythrocytes were observed, which can be characterized as late metestrous and diestrous stages (Fig. 1A). Changes in the estrous cycle after repeated injections of gonadotropins were summarized in a dot graph. The estrous cycle tended to shift from estrous to diestrous stages after repeated stimulation of gonadotropins (Fig. 1B). As a result of observing the reproductive organs with the naked eye after opening the abdomen, the ovaries and uterus swelled and blood vessels developed in both the OS and $\mathrm{RS}$ groups, but there were no noticeable morphological differences between the two groups (Fig. $1 \mathrm{C})$.

\section{Effect of repeated ovarian stimulation on ovarian follicle development}

The ovaries were stained with Hematoxylin and eosin, and follicles at each stage of folliculogenesis were counted to evaluate the effect of repeated ovarian stimulation on follicular development. In the OS group, the proportion of primary follicles (PF) was $65 \%$, secondary follicles (SF) 8\%, antral follicles (AF) 9\%, Graffian follicles (GF) 3\%, and corpus luteum (CL) 14\%. In the RS group, the proportion of $\mathrm{PF}$ was $27 \%$, SF $13 \%, \mathrm{AF} 16 \%$, GF 3\%, and CL 41\%. The proportion of $\mathrm{PF}$ in the RS group was significantly reduced from $65 \%$ to $27 \%$ compared to the OS group. In contrast, the proportion of CL was significantly increased in the RS group compared to the OS group. On the other hand, the proportion of SF, AF, and GF had no difference between the two groups (Fig. 2A, B).

\section{Effect of repeated ovarian stimulation on apoptosis in the ovaries}

TUNEL staining was performed to detect apoptotic cells in the ovaries. In the OS group, apoptotic cells were detected in $18 \%$ of $\mathrm{AF}, 50 \%$ of GF, and $17 \%$ of CL. In the RS group, apoptotic cells were detected in $23 \%$ of $\mathrm{AF}$ and $42 \%$ of GF but not in CL. The rate of apoptosis during folliculogenesis did not differ between the two groups, but apoptosis in CL was significantly reduced in the RS group (Fig. 3A, B).

\section{Effect of repeated ovarian stimulation on the expression of apoptosis-related genes}

To elucidate the effect of repeated ovarian stimulation on the expression of apoptosis-related genes apoptosis in the ovaries, $m T O R$, Fas, and Fas $L$ mRNA were detected by qRT-PCR. There was no significant difference in $m T O R$, Fas, and FasL mRNA expression levels when OS and RS groups were compared (Fig. 4). 
A.

B.
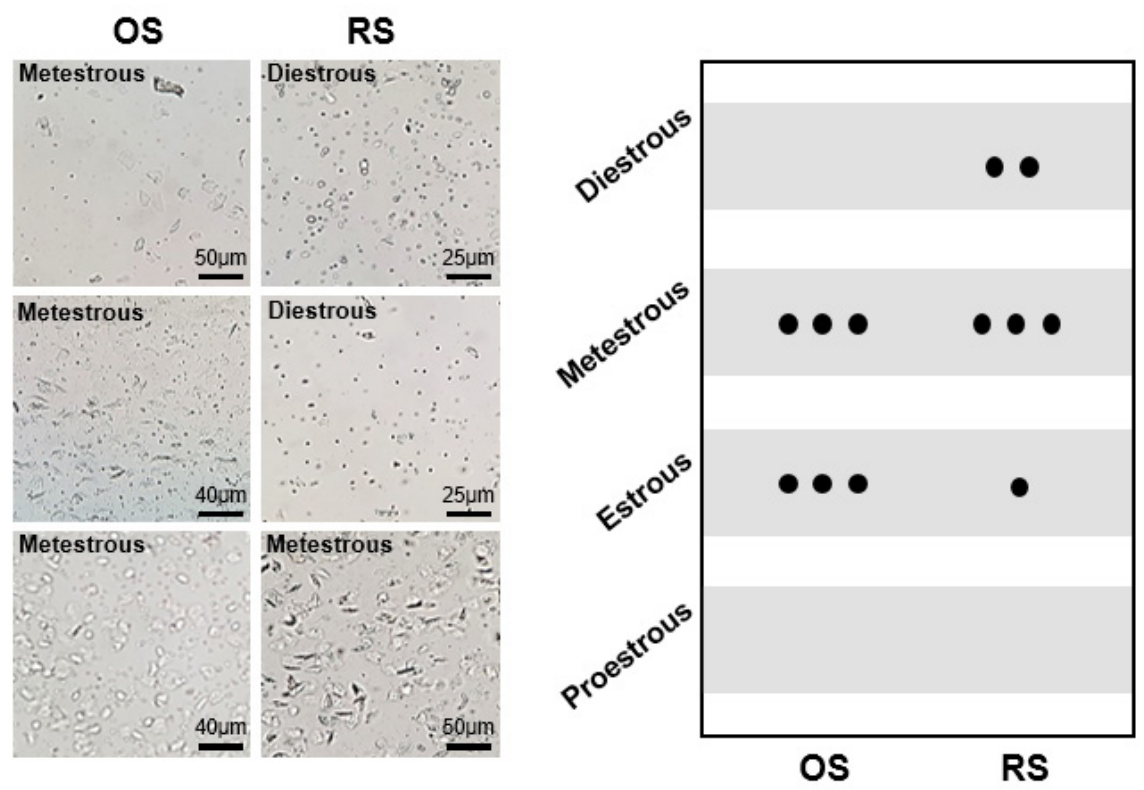

C.
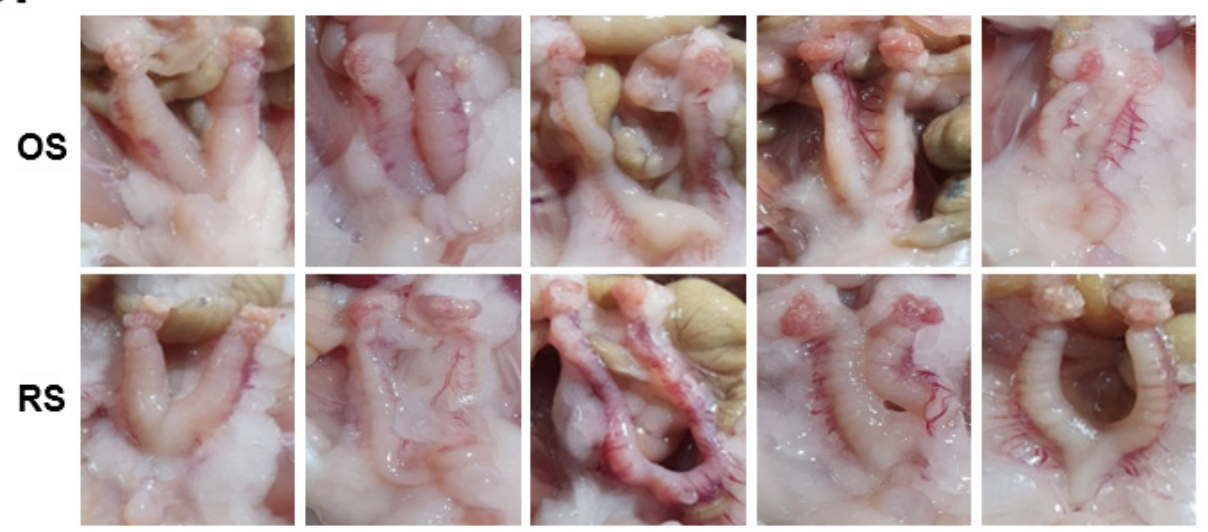

Fig. 1. The effect of repeated ovarian stimulation on estrous cycle in mice ovaries. (A) The smear test was used to examine endometrial cells in OS and RS group mice. (B) The smear test validated the estrous cycle status of OS $(n=6)$ and RS $(n=6)$ groups mice. (C) Visual inspection was used to examine morphological changes in the uterus and ovaries of mice in OS and RS group mice. OS, once stimulation; RS, repeated stimulation.

\section{Effect of repeated ovarian stimulation on the expression of autophagy-related genes}

To elucidate the effect of repeated ovarian stimulation on the expression of autophagy-related genes in the ovaries, Atg5, Atg12, LC3B, and Beclin1 mRNA was detected by qRT-PCR. There was no significant difference in Atg5, Atg12, LC3B, and Beclin1 mRNA expression levels when OS and RS groups were compared (Fig. 5).

\section{Effect of repeated ovarian stimulation on the expression of aging-related genes}

To elucidate the effect of repeated ovarian stimulation on the expression of aging-related genes in the ovaries, Dnmt1, Dnmt3a, and $A M H$ mRNA was detected by qRT-PCR. There was no 
A.

OS

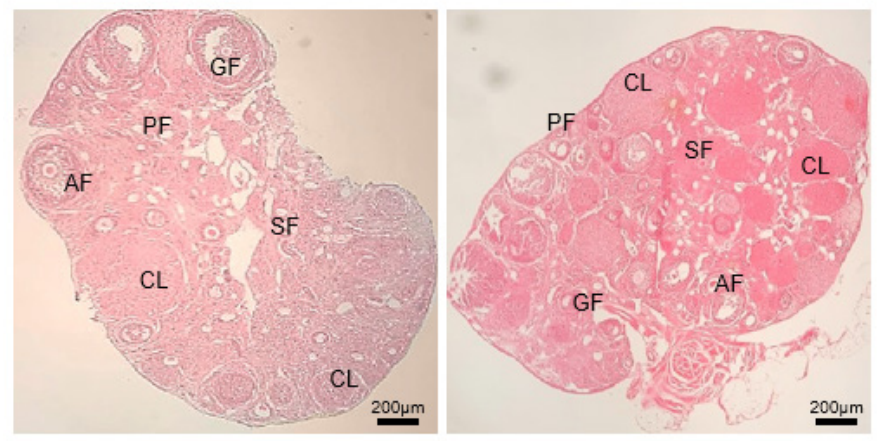

B.

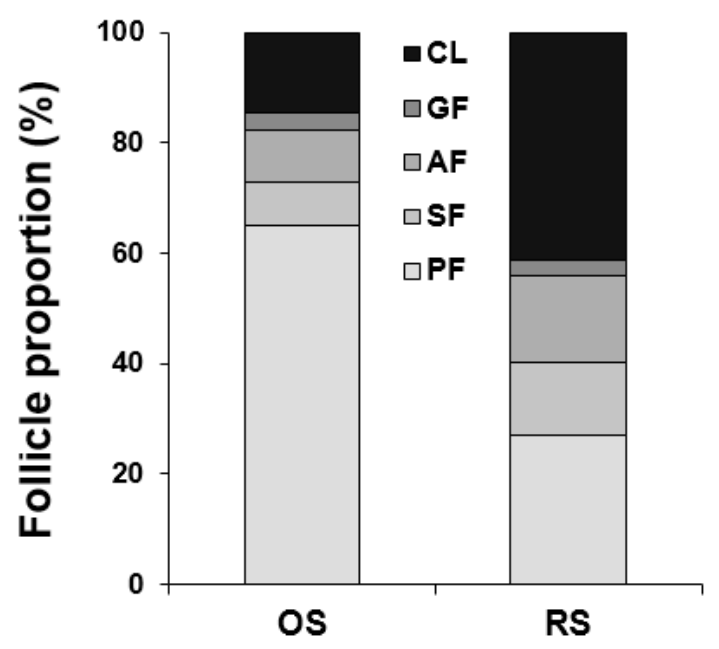

Fig. 2. The effect of repeated ovarian stimulation on follicle development in mice ovary. (A) Ovarian sections from OS and RS group mice stained with hematoxylin \& eosin. (B) Comparison of follicle ratio at each stage of follicular development by repeated stimulation. The proportion of PF has significantly decreased, whereas the proportion of $\mathrm{CL}$ has significantly increased. OS, once stimulation; RS, repeated stimulation; $\mathrm{CL}$, corpus luteum; GF, Graffian follicle; AF, antral follicle; SF, secondary follicle; PF, primary follicle.

A.

OS
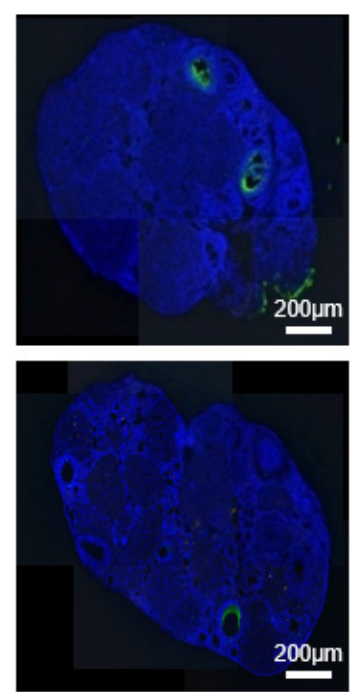

RS
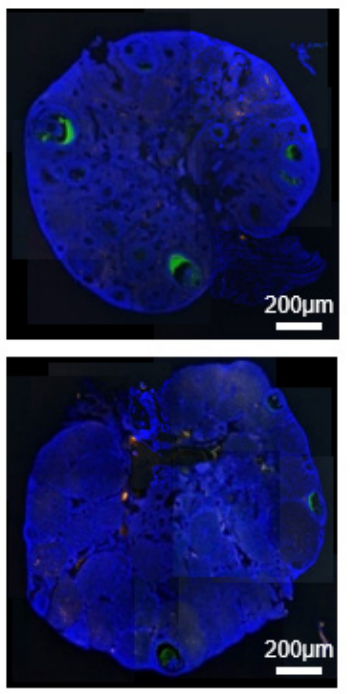

B.

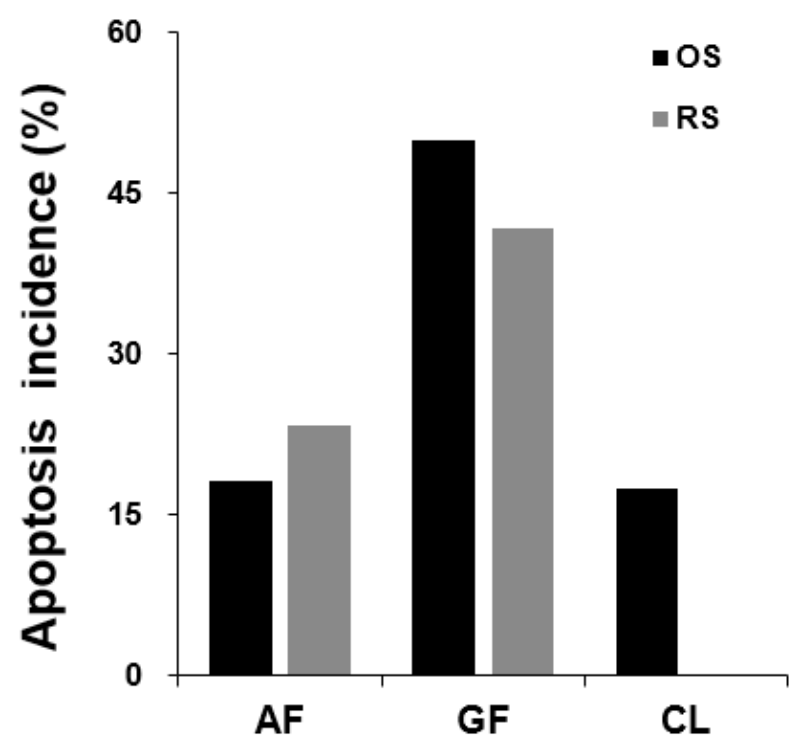

Fig. 3. The effect of repeated ovarian stimulation on mouse ovarian apoptosis. (A) TUNEL assay was used to confirm the occurrence of apoptosis in OS and RS group mice ovaries. Apoptosis is indicated by the presence of green fluorescence. (B) The number of follicles with apoptosis as a percentage of the total number of follicles was used to calculate the incidence of apoptosis. Apoptosis was only confirmed in the antral follicle, Graffian follicle, and corpus luteum in the OS group. Only the antral and Graffian follicles in the RS group showed signals of apoptosis. No apoptosis occurred in corpus luteum of RS group. OS, once stimulation; RS, repeated stimulation; AF, antral follicle; GF, Graffian follicle, CL, corpus luteum. 

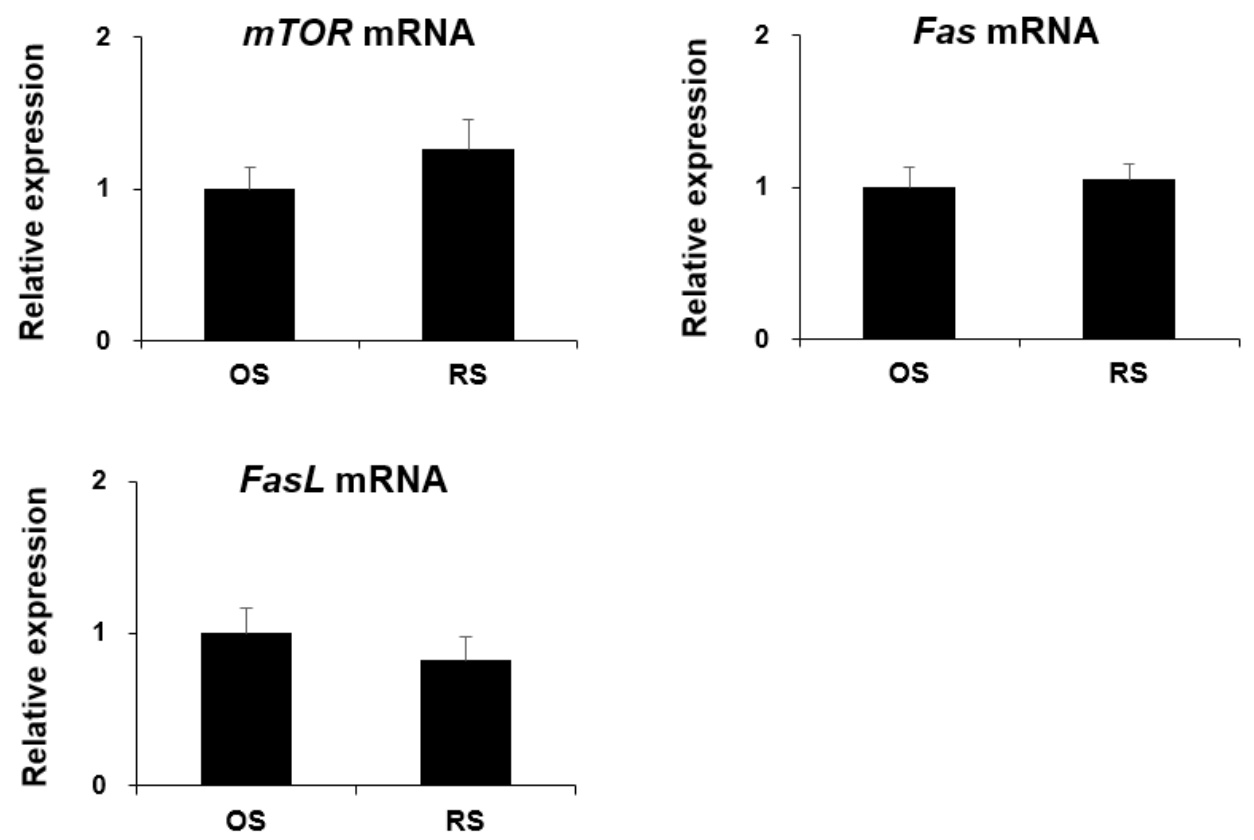

Fig. 4. The effect of repeated ovarian stimulation on apoptosis-related gene mRNA expression. $m T O R$, Fas, and FasL were used as ovarian apoptosis-related genes. mRNA expression levels were confirmed and compared through quantitative RT-PCR. There was no significant change in mRNA expression levels of $m T O R$, Fas, and FasL genes. All data are represented as means \pm SEM. OS, once stimulation; $\mathrm{RS}$, repeated stimulation.
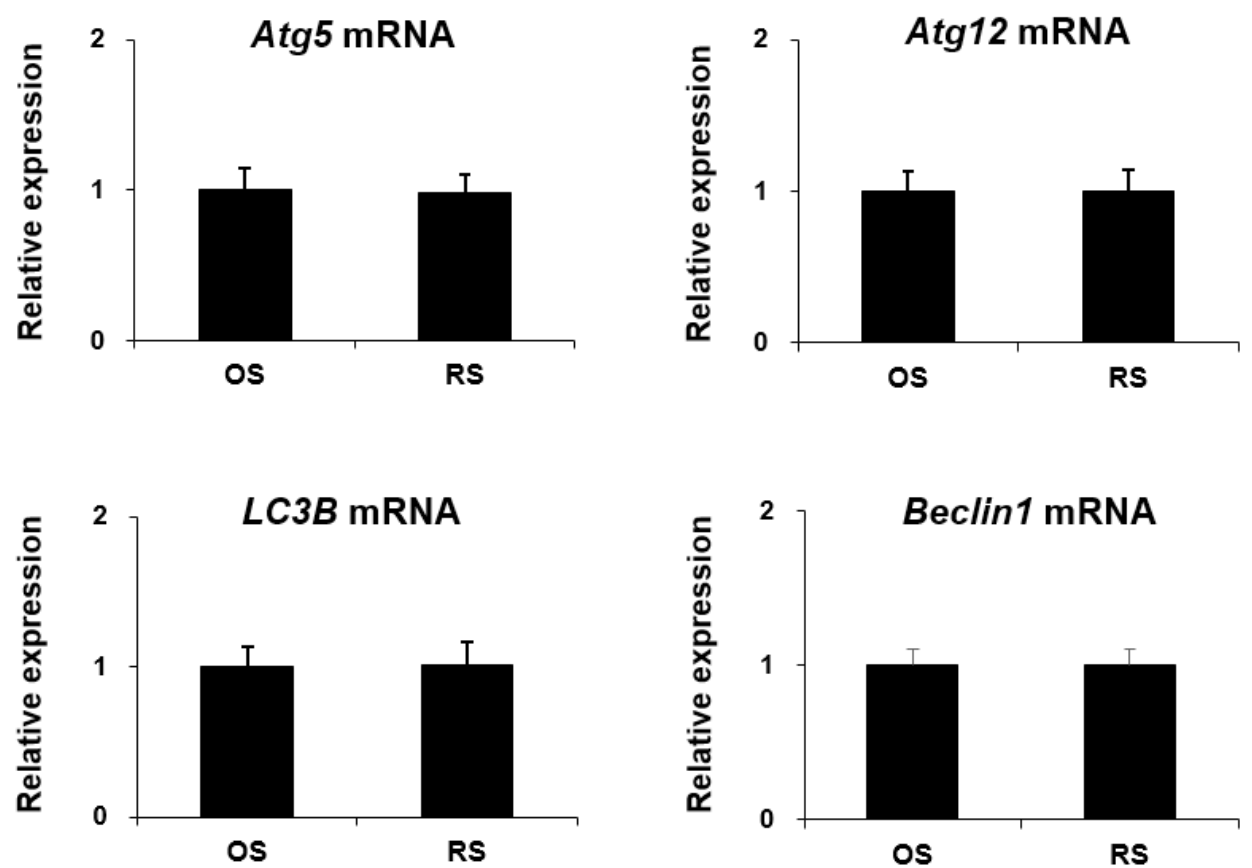

Fig. 5. The effect of repeated ovarian stimulation on autophagy-related gene mRNA expression. Atg5, Atg12, LC3B, and Beclin1 were used as ovarian autophagy-related genes. mRNA expression levels were confirmed and compared through quantitative RT-PCR. There was no significant change in mRNA expression levels of Atg5, Atg12, LC3B, and Beclin1 genes. All data are represented as means \pm SEM. OS, once stimulation; RS, repeated stimulation. 
significant difference in Dnmt1, Dnmt3a, and $A M H$ mRNA expression levels when OS and RS groups were compared (Fig. 6).

\section{DISCUSSION}

The $\mathrm{COH}$ is the first step in the IVF-ET process to collect many good-quality oocytes. The patient receives repeated injections of large doses of gonadotropins during the $\mathrm{COH}$. However, this $\mathrm{COH}$ repeated ovarian stimulation might lead to problems with ovarian follicle development. Therefore, we investigated follicular development, follicular atresia involving apoptosis and autophagy, and ovarian aging-related genes in mouse ovaries after repeated stimulation with gonadotropins.

A vaginal smear test was performed to investigate the effect of repeated ovarian stimulation on the estrous cycle. The estrous cycle stage was identified by observing the cells obtained from vaginal flushing. After one ovarian stimulation with PMSG and hCG, the estrous cycle was in estrous and metestrous stages. On the other hand, the estrous cycle was metestrous and diestrous stages after repeated ovarian stimulation. These results showed that repeated ovarian stimulation prolongs the luteal phage in which the CL develops. A recent report showed that repeated ovarian stimulation at 16-day intervals alters the estrus cycle, inducing uterine cell proliferation in mice (Antonouli et al., 2020). In monkeys, however, observations of the uterus after 5 years of repeated ovarian stimulation did not result in significant changes in the endometrium (Yan et al., 2017). These results suggest that ovarian hyperstimulation at short intervals may also cause problems with uterine function, but not at long intervals.

To assess ovarian follicle development after ovarian stimulation, the number of follicles at each
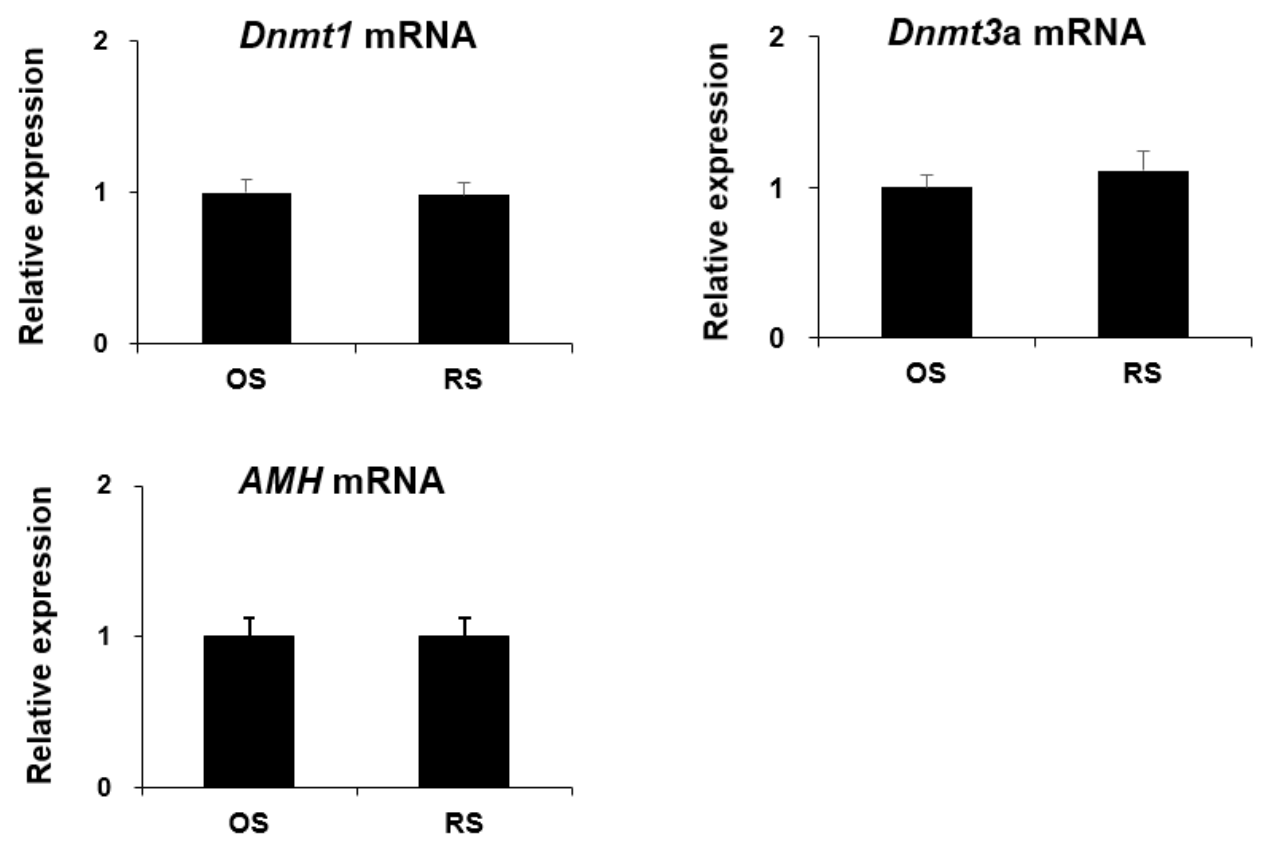

Fig. 6. The effect of repeated ovarian stimulation on aging-related gene mRNA expression. Dnmt1, Dnmt3a, and $A M H$ were used as ovarian aging-related genes. mRNA expression levels were confirmed and compared through quantitative RT-PCR. There was no significant change in mRNA expression levels of Dnmt1, Dnmt3a, and AMH genes. All data are represented as means \pm SEM. OS, once stimulation; $\mathrm{RS}$, repeated stimulation. 
stage was counted in $\mathrm{H} \& \mathrm{E}$-stained ovarian sections. In the OS group, $\mathrm{PF}$ accounted for the most significant proportion, while the CL proportion was as low as $17 \%$. Conversely, the CL accounted for the most significant proportion in the RS group, and the number of secondary follicles and antral follicles was also increased. However, the PF proportion was as low as $24 \%$ compared to the OS group. These results suggest that repeated injection with gonadotropins promotes follicular development and activates the luteal phase of the estrous cycle for a long time. Ovarian hyperstimulation with gonadotropins is known to promote follicular development, which promotes the growth and development of preovulatory follicles and induces premature luteinization (Chaffin \& Stouffer, 2000). It has been reported that repeated ovarian hyperstimulation prolongs the luteal phase and decreases the number of primary and secondary follicles while increasing the number of atretic follicles in mouse ovaries (Wang et al., 2003; Nie et al., 2018).

Given the changes in folliculogenesis after repeated injections of gonadotropins, we assumed that repeated ovarian stimulation might induce apoptosis in the ovaries. It is well known that apoptosis is associated with follicle atresia, which reduces the number of follicles that grow and ovulate in the ovary (Santos et al., 2010). There, we examined whether repeated ovarian stimulation affects the apoptosis of granulosa cells in follicles by TUNEL assay. The rate of apoptosis in the ovaries after repeated ovarian stimulation did not differ between the two groups. It is reported that repeated ovarian stimulation induces oxidative stress in the ovary, and the accumulation of this oxidative stress promotes follicle depletion (Chao et al., 2005; Dong et al., 2014). Moreover, number of apoptotic cells in the ovarian follicles increases within 24 hours after hCG administration. The increased apoptosis in ovarian follicles leads to follicular atresia and decreased ovarian function (Yu et al., 2004; Nie et al., 2018). In this study, however, we demonstrated that repeated ovarian stimulation did not affect follicular development and atresia.

Next, we investigated the expression levels of apoptosis-related genes such as mTOR, Fas, and FasL in the ovaries after repeated ovarian stimulation. Fas is a cell-surface death receptor with an intracellular death domain that initiates apoptosis (Nagata, 1998). The binding of Fas-ligand to Fas activates Fas-associated protein with death domain (FADD) and sub-factors such as caspase 8, caspase 3, and caspase 7 to induce apoptosis (Kaufmann et al., 2012). On the other hand, $m$ TOR is well known to be involved in cell proliferation and survival (Castedo et al., 2002; Zou et al., 2020). Our results showed no significant difference in $m T O R$, Fas, and Fas $L$ mRNA expression levels between OS and RS groups, similar to TUNEL assay results. These results suggest that repeated gonadotropins injection does not affect apoptosis in the ovary. We also evaluated the expression levels of autophagy-related genes such as Atg5, Atg12, LC3B, and Beclin1. Generally, it is well known that autophagy maintains homeostasis and is involved in cell survival, but induces apoptosis when stress stimulates cells. In the ovary, autophagy plays an important role in cell survival from PFs to preantral follicles or as a mechanism for apoptosis in closed follicles and degenerating CL (Leopardo et al., 2020). The key genes involved in the autophagy are Atg5, Atg12, LC3B, and Beclin1. Their mRNA expression levels were not significantly different between OS and RS groups, similar to the expression of apoptosis-related genes. Finally, to determine whether repeated ovarian stimulation affects ovarian aging, we detected aging-related genes such as Dnmt1 and Dnmt3a. These are DNA methyltransferases that promote aging by inducing gene methylation, and are representative markers of aging (Xi et al., 2019). Dnmt1 and Dnmt3a reduce autophagy activity by upregulating the methylation of $A \operatorname{tg} 5, A \operatorname{tg} 12$, and $L c 3 B$ genes, which are autophagy-related genes (Li et al., 2020). The expression level of aging-related genes did not differ between the two groups. These results suggest that repeated gonadotropins injection does not affect autophagy and aging in the ovary.

In summary, repeated ovarian stimulation with PMSG and hCG resulted in prolonged luteal 
phase in the estrous cycle in mice. After ovarian hyperstimulation, the proportion of CL increased while the proportion of primary and secondary follicles decreased. However, there was no change in follicular apoptosis by repeated ovarian stimulation. Moreover, there was no change in the expression of genes associated with apoptosis, autophagy, and aging by repeated ovarian stimulation. Taken together, these results suggest that repeated ovarian stimulation with gonadotropins during IVF-ET procedures may not cause problems such as excessive follicular atresia or ovarian aging.

\section{REFERENCES}

Acevedo B, Gomez-Palomares JL, Ricciarelli E, Hernández ER (2006) Triggering ovulation with gonadotropin-releasing hormone agonists does not compromise embryo implantation rates. Fertil Steril 86:1682-1687.

Antonouli S, Palmerini MG, Bianchi S, Rossi G, Cecconi S, Belli M, Bernardi S, Khalili MA, Familiari G, Nottola SA, Macchiarelli G (2020) Repeated hyperstimulation affects the ultrastructure of mouse fallopian tube epithelium. J Reprod Dev 66:387-397.

Baker TG (1963) A quantitative and cytological study of germ cells in human ovaries. Proc R Soc Lond B Biol Sci 158:417-433.

Caligara C, Navarro J, Vargas G, Simón C, Pellicer A, Remohí J (2001) The effect of repeated controlled ovarian stimulation in donors. Hum Reprod 16:2320-2323.

Castedo M, Ferri KF, Kroemer G (2002) Mammalian target of rapamycin (mTOR): Pro- and antiapoptotic. Cell Death Differ 9:99-100.

Chaffin CL, Stouffer RL (2000) Role of gonadotrophins and progesterone in the regulation of morphological remodelling and atresia in the monkey peri-ovulatory follicle. Hum Reprod 15:2489-2495.

Chao HT, Lee SY, Lee HM, Liao TL, Wei YH, Kao SH (2005) Repeated ovarian stimulations induce oxidative damage and mitochondrial DNA mutations in mouse ovaries. Ann NY Acad Sci 1042:148-156.

Chari T, Griswold S, Andrews NA, Fagiolini M (2020) The stage of the estrus cycle is critical for interpretation of female mouse social interaction behavior. Front Behav Neurosci 14:113.

Christenson LK, Stouffer RL (1997) Follicle-stimulating hormone and luteinizing hormone/ chorionic gonadotropin stimulation of vascular endothelial growth factor production by macaque granulosa cells from pre- and periovulatory follicles. J Clin Endocrinol Metab 82:2135-2142.

Dong G, Guo Y, Cao H, Zhou T, Zhou Z, Sha J, Guo X, Zhu H (2014) Long-term effects of repeated superovulation on ovarian structure and function in rhesus monkeys. Fertil Steril 102:1452-1457.

Griesinger G (2010) Ovarian hyperstimulation syndrome prevention strategies: Use of gonadotropin-releasing hormone antagonists. Semin Reprod Med 28:493-499.

Hansen KR, Knowlton NS, Thyer AC, Charleston JS, Soules MR, Klein NA (2008) A new model of reproductive aging: The decline in ovarian non-growing follicle number from birth to menopause. Hum Reprod 23:699-708.

Jacobs HS, Agrawal R (1998) 4 Complications of ovarian stimulation. Baillieres Clin Obstet Gynaecol 12:565-579.

Kaufmann T, Strasser A, Jost PJ (2012) Fas death receptor signalling: Roles of Bid and XIAP. Cell Death Differ 19:42-50.

Leopardo NP, Velazquez ME, Cortasa S, González CR, Vitullo AD (2020) A dual death/survival 
role of autophagy in the adult ovary of Lagostomus maximus (Mammalia-Rodentia). PLOS ONE 15:e0232819.

Li P, Zhu H, Tan L, Zhao D, Ma L, Xiang Y, Zhang D, Dou Q, Lu N (2015) Effects of high progesterone on outcomes of in vitro fertilization-embryo transfer in patients with different ovarian responses. Syst Biol Reprod Med 61:161-167.

Li Q, Cai M, Wang J, Gao Q, Guo X, Jia X, Xu S, Zhu H (2020) Decreased ovarian function and autophagy gene methylation in aging rats.J Ovarian Res 13:12.

Macklon NS, Stouffer RL, Giudice LC, Fauser BCJM (2006) The science behind 25 years of ovarian stimulation for in vitro fertilization. Endocr Rev 27:170-207.

Nagata S (1998) Fas-induced apoptosis. Intern Med 37:179-181.

Nie X, Dai Y, Zheng Y, Bao D, Chen Q, Yin Y, Fu H, Hou D (2018) Establishment of a mouse model of premature ovarian failure using consecutive superovulation. Cell Physiol Biochem 51:2341-2358.

Santos MA, Kuijk EW, Macklon NS (2010) The impact of ovarian stimulation for IVF on the developing embryo. Reproduction 139:23-34.

Wang Z, Tamura K, Yoshie M, Tamura H, Imakawa K, Kogo H (2003) Prostaglandin F ${ }_{2 \alpha}$-induced functional regression of the corpus luteum and apoptosis in rodents.J Pharmacol Sci 92:19-27.

Xi X, Zou Q, Wei Y, Chen Y, Wang X, Lv D, Li P, Wen A, Zhu L, Tang G, Ma J, Li M, Jiang Y (2019) Dynamic changes of DNA methylation and transcriptome expression in porcine ovaries during aging. BioMed Res Int 2019:8732023.

Yan P, Xu J, Zeng Y, Dong G, Cao H, Zheng M, Zhu H (2017) Long-term effects of repeated superovulation on the uterus and mammary gland in rhesus monkeys. J Assist Reprod Genet 34:535-545.

Yu YS, Sui HS, Han ZB, Li W, Luo MJ, Tan JH (2004) Apoptosis in granulosa cells during follicular atresia: Relationship with steroids and insulin-like growth factors. Cell Res 14:341346.

Zou Z, Tao T, Li H, Zhu X (2020) mTOR signaling pathway and mTOR inhibitors in cancer: Progress and challenges. Cell Biosci 10:31. 
